\title{
Challenges in Retention of Patients in Continuum of HIV-Care in Delhi- Experience of a Decade \& Way Ahead
}

\author{
Anil Kumar Gupta1*, Vandana Dabla1, Bipin Chandra Joshi'1, Sabyasachi Chakraborty1, \\ Jiban Jyoti Baishya ${ }^{2}$, Abhinav Gupta ${ }^{1}$ \\ ${ }^{1}$ Delhi State AIDS Control Society, Government of NCT of Delhi, New Delhi, India \\ ${ }^{2}$ National AIDS Control Organization, Ministry of Health \& Family Welfare, Government of India, New Delhi, India \\ Email: dr.a.k.gupta@gmail.com, vandana.dabla@gmail.com, bipincjoshi007@gmail.com, \\ arjunsabya@gmail.com, rc.naco.dmpc@gmail.com, abhinav491@gmail.com
}

Received 4 September 2014; revised 4 October 2014; accepted 1 November 2014

Copyright (C) 2014 by authors and Scientific Research Publishing Inc.

This work is licensed under the Creative Commons Attribution International License (CC BY). http://creativecommons.org/licenses/by/4.0/

(c) (i) Open Access

\section{Abstract}

Retention of the patients in HIV-care is critical for success of Anti Retroviral Treatment (ART) programme to reduce HIV-related morbidity \& mortality and prevent emergence of drug resistance. In last decade in Delhi (April 2004 to March 2014), overall 24\% HIV-positive patients were lost-to-follow-up (LTFU) at step-1 (testing to enrolment into HIV-care), $7.8 \%$ at step-2 (enrolment to ART eligibility), $23.7 \%$ at step-3 (eligibility to initiation of ART) and $16.6 \%$ at step-4 (initiation to lifelong ART) of retention cascade. About $2 / 3$ rd losses at step-4 were within 1st year and $80 \%$ within 2 years. The retention of the patients in pre-ART care was 3 times lower than those initiated ART. Only $27.4 \%$ patients were in active pre-ART care during 2013 . The intensified LTFU tracking (ILT) undertaken during November, 2013 through March, 2014 was not successful in tracking $97 \%$ pre-ART LTFU clients due to incomplete addresses/or migration since address proof of patients on enrolment into HIV-care was not mandatory prior to 2009. Amongst patients tracked, $1.5 \%$ were alive, $0.24 \%$ had disengaged from care while $1.2 \%$ had died. After ILT overall "On ART" and "Pre-ART" LTFU rate in the last decade was $15.5 \%$ and $45.2 \%$, respectively. The retention cascade of last year from April 2013 to March 2014 showed improvement through strategies adopted in Third Phase of National AIDS Control Programme (NACP-III; 2007-2013), and "On ART" and "Pre-ART" LTFU rates declined to $9.4 \%$ and $7.4 \%$, respectively. However, desired at least $\mathbf{9 0 \%}$ retention at various steps of the cascade could not be achieved. National Policy of delivering ART services through limited number of standalone ART centers in India, despite its significant success, has limitation of leaky treatment cascade and calls for policy makers to decentralize the programme by its appropriate integration with general health services and task shifting to improve continuum of care.

\footnotetext{
${ }^{*}$ Corresponding author.
}

How to cite this paper: Gupta, A.K., Dabla, V., Joshi, B.C., Chakraborty, S., Baishya, J.J. and Gupta, A. (2014) Challenges in Retention of Patients in Continuum of HIV-Care in Delhi-Experience of a Decade \& Way Ahead. World Journal of AIDS, 4, 387-395. http://dx.doi.org/10.4236/wja.2014.44046 


\section{Keywords}

\section{Retention in HIV-Care, Pre-ART Care, Continuum of Care, Antiretroviral Treatment, Decentralization}

\section{Introduction}

Retention of patients in HIV-care is vital for success of Anti Retroviral Treatment (ART) programme to reduce HIV-related morbidity \& mortality and prevent emergence of drug resistance [1]. People Living with HIV/AIDS (PLHIV) may be lost at any of four steps of treatment cascade, first, HIV testing to enrolment into care services, second, enrolment in care to ART eligibility, third, eligibility to initiation of ART, and fourth, initiation to lifelong ART [1]. This study describes challenges to retain PLHIV in HIV-care during last decade of ART programme in Delhi. National Policy of delivering ART services through limited number of standalone ART centers in India, despite success, has limitation of leaky treatment cascade [2] and calls for policy makers to follow latest WHO recommendations [3] of decentralizing ART programme by its appropriate integration with general health services and task shifting to improve continuum of care during fourth phase of the National AIDS Control Programme (NACP-IV).

\section{Material \& Methods}

\subsection{State Profile}

Delhi is a low HIV prevalence state of India with $0.3 \%$ prevalence in general population against national prevalence of $0.27 \%$. Over the years, HIV prevalence has shown a steady decline in high risk groups (HRGs) and general population [4].

\subsection{Scale up of Services under NACP-III (2007-2013)}

ART programme was rolled out in 2004 and rapidly scaled up by establishing 9 ART centers across Delhi. Access to HIV counseling and testing services was scaled up in NACP-III (2007-2013). Currently 89 standalone, 72 facility-integrated and 3 mobile Integrated Counseling \& Testing Centres (ICTCs) are functional in Delhi. Five Community Care Centers (CCCs) were established for out-patients and in-patient care of PLHIV, drug adherence counseling, and tracking cases lost-to-follow-up (LTFU). Communities of PLHIV were mobilized to support PLHIV through advocacy to facilitate delivery of right based HIV-care and treatment. In April, 2013 CCCs run by NGOs were discontinued by NACO and replaced by 2 Community Support Centres (CSCs) and 5 PLHIV help desks to enhance community support in programme [2].

\subsection{Eligibility Criteria for Initiation of ART}

As per National Guidelines, HIV-positive patients with CD4-count $\leq 350$ cells/cu.mm, patients in WHO clinical stage III/IV or with HIV-TB co-infection, are initiated lifelong ART [5]. In January, 2014 country has adopted Option B+ strategy for prevention of mother to-child HIV transmission, wherein HIV-positive pregnant women are initiated lifelong ART at earliest opportunity in antenatal period, irrespective of CD4-count [6].

\subsection{CD4-Test Facility}

Delhi has 5 CD4-testing sites for 9 ART centres. Only 4 CD4 sites are co-located with ART centres. In centers where there is no CD4 machine, a linkage has been established with a nearby CD4 site whereby blood samples are collected and transported by ART Lab Technician and patient does not have to go to another site to get CD4test done.

\subsection{Supply Chain Management}

Anti Retroviral (ARV) medicines and CD4-test kits are centrally procured by NACO and supplied to DSACS. 
ARV medicines are supplied by DSACS to the ART centres on the basis of quarterly consumption.

\subsection{Retention Cascade}

Once an ICTC attendee is detected HIV-positive, patient is referred after post-test counseling to nearest ART centre for registration in HIV-care. Proof of address of patient at time of ART registration was made mandatory in 2009. Each patient registered in HIV-care undergoes counseling, clinical examination and baseline investigations, including CD4-test. If the patient is eligible for ART [5], lifelong ART is initiated after at least 2 sessions of counseling. ARV medicines are provided to patients initially for 15 days and thereafter every month for up-to 12 months to ensure regular visit of patient to ART centre for clinical examination and ensuring drug adherence through self assessment of patient and pill count. CD4-count is performed every 6 months after initiating ART, unless clinically indicated early. After completion of 12 months of treatment, if clinical and immunological response is appropriate, patient is subsequently provided ARV drugs for every 2 months. Patients suspected for treatment failure are referred to Centre of Excellence for ART for 2nd line treatment [5]. Patients not eligible for ART continue in Pre-ART care and are required to return to ART centre every 6 months for CD4-count or early in event of any opportunistic infection (O.I.) Daily due lists for clinical visit and CD4-count is prepared by ART centres. Any patient who miss visit to ART centre on due date is first contacted telephonically by the ART counselor followed by home visit by ORW, if required. Patient who fail to visit ART centre for 90 days or longer after last given appointment is labeled as LTFU. List of LTFU cases is provided to ORWs for home visits of patients. Monthly meetings are conducted to review cases tracked.

\subsection{Recording \& Reporting}

ART centers maintain records of patients in pre-ART and ART enrollment registers. Master line lists are prepared by each ART centre in Computerized Management Information System (CMIS) Software. ART centres send monthly reports to DSACS electronically.

\subsection{Study Design}

CMIS data of ART programme was analyzed for period from 1st April, 2004 through 31st March, 2014 for following indicators:

1) Cumulative number of ICTC clients detected HIV-positive;

2) Cumulative number of HIV-positive patients registered in HIV-care;

3) Out of 2) number of patients undergone CD4-test;

4) Out of 3) number of patients eligible for ART;

5) Out of 4) number of patients initiated ART;

6) Out of 5) number of patients alive on ART, or died, or transferred out, or LTFU, or opted out of the programme.

\subsection{Intensified LTFU Tracking (ILT)}

On directions of NACO, an Intensified LTFU Tracking (ILT) drive was rolled out in November, 2013 to retrieve patients LTFU from ART and Pre-ART care. ART centres were instructed to send master line-lists from PreART and ART enrolments registers up-to 31st March, 2014 to DSACS. List for Pre-ART and "On ART” LTFU cases was prepared and shared with 4 District AIDS Prevention Control Units (DAPCUs) with instruction to pool-in ORWs from different components of HIV programme and divide and plot 9 districts among them evenly. Home visits by outreach teams were undertaken during November, 2013 through March, 2014. Outcome of Pre-ART and On ART LTFU cases was evaluated.

The term active pre-ART care was used for patients in pre-ART care who had undergone 6 monthly CD4count in 2013. The term LTFU was used for patients with unknown outcomes while patients who ceased to engage in continuum of care because of their wishes/or beliefs were labeled as "disengaged from care".

\section{Results}

Decadal retention cascade of Delhi is presented in Figure 1. As evident, 13,393 (24\%) HIV-positive patients got 


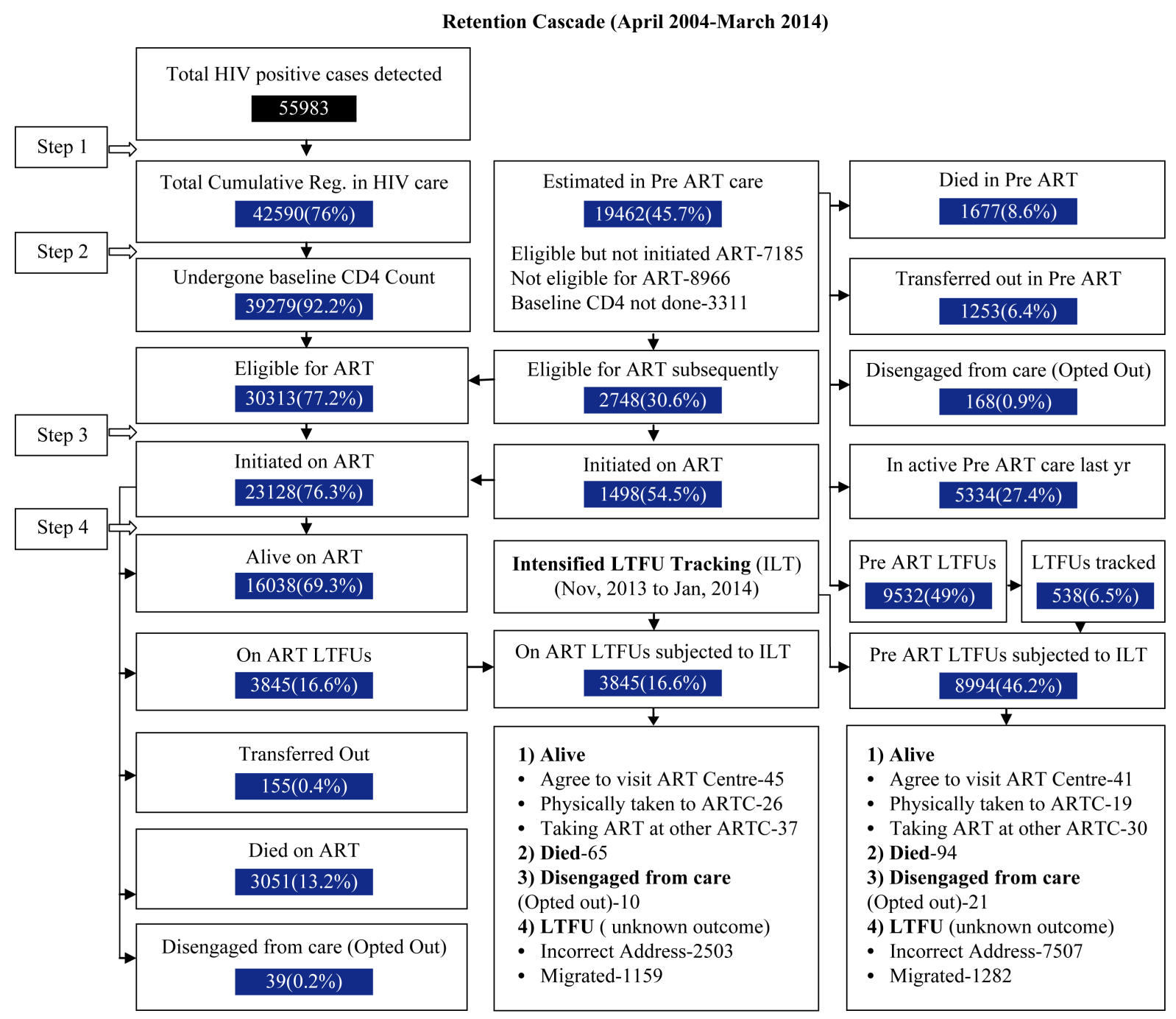

Figure 1. Decadal retention cascade of Delhi (April 2004-March 2014).

LTFU at step-I (HIV testing to enrolment into care services), 3311 (7.8\%) at step 2 (enrolment in care to ART eligibility), 7185 (23.7\%) at step 3 (eligibility to initiation of ART) and 3845 (16.6\%) at step 4 (initiation to lifelong ART) of cascade. Table 1 shows duration of ART in “On ART LTFU” cases before LTFU. As evident, 23\% patients did not return to ART clinic after initiation of ART. About 2/3rd (67\%) losses at step 4 were within 1 year of initiation of ART and $80 \%$ within 2 years.

Amongst 19,462 patients in Pre-ART care, 7185 (37\%) were eligible but not initiated ART, 8966 (46\%) were not eligible for ART at time of registration in HIV-care while 3311 (16\%) did not undergo CD4-count. However, only 5334 (27.4\%) cases were in active pre-ART care in 2013. After excluding deaths, cases transferred out to other states or disengaged from care or in active pre-ART care, 8994 (46.2\%) cases were recorded as LTFU. The yearly-trend of pre-ART LTFU is presented in Figure 2. As evident, number of cases LTFU in pre-ART care declined from 7327 (79.3\%) during 2007-2009 to 1667 (16.3\%) during 2010-2013.

ILT was undertaken during Nov, 2013 through Jan, 2014 by home visits of 12839 LTFU patients. 198 (1.5\%) patients were found alive, 159 (1.2\%) had died, 31 (0.24\%) had disengaged from care while outcome of 12,451 (97\%) patients was unknown due to incomplete/incorrect addresses or migration. After ILT, overall “On ART” and "Pre-ART" LTFU rates of last decade were $15.5 \%$ and $45.2 \%$, respectively and overall retention at 4 steps of the decadal cascade after excluding deaths, official transfer-outs, disengaged from care was $76 \%, 92.2 \%$, $76.3 \%$ and $81.2 \%$, respectively. 


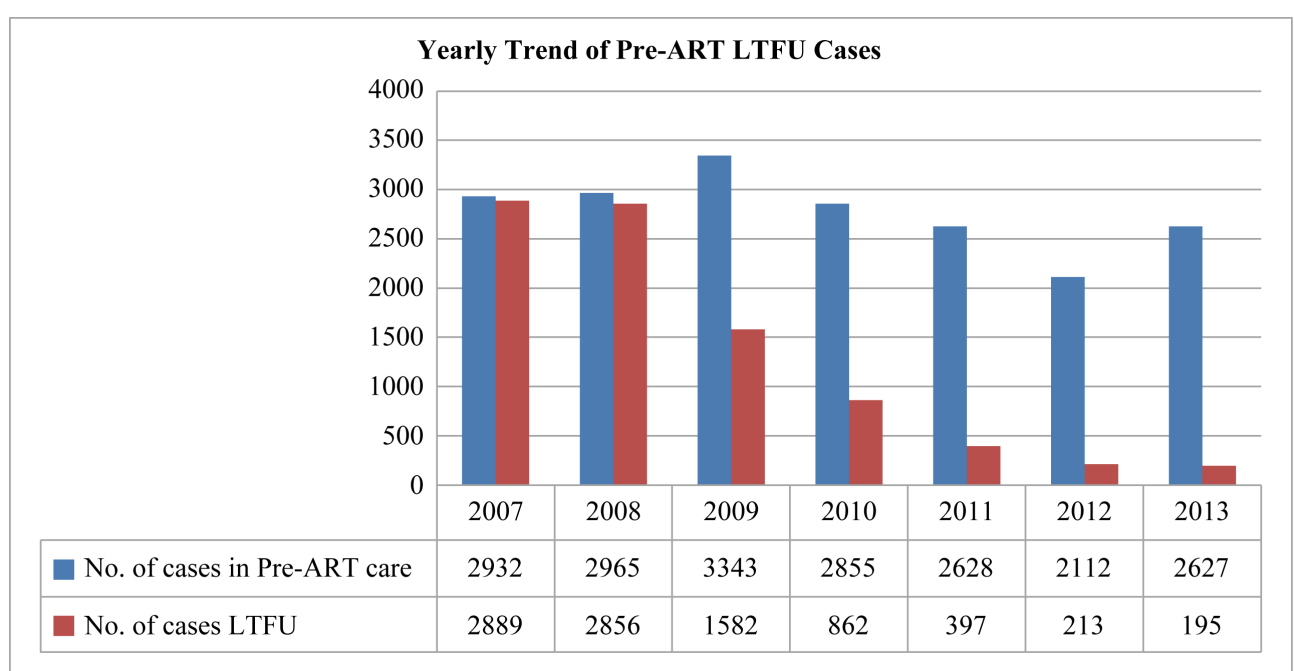

Figure 2. Yearly trend of Pre-ART LTFU cases during NACP-III in Delhi (April 2007-March 2014).

Table 1. Showing duration of ART in patients prior to LTFU $(n=3845)$.

\begin{tabular}{ccc}
\hline Duration of ART (months) & Number & $\%$ \\
\hline 0 to 0.5 & 399 & 10.4 \\
1 & 486 & 12.6 \\
2 & 353 & 9.2 \\
3 to 6 & 800 & 20.8 \\
7 to 12 & 551 & 14.3 \\
13 to 24 & 503 & 13.1 \\
25 to 36 & 276 & 7.2 \\
37 to 48 & 189 & 4.9 \\
49 to 60 & 81 & 2.1 \\
61 to 72 & 81 & 2.1 \\
73 to 84 & 63 & 1.6 \\
85 to 96 & 42 & 1.1 \\
97 to 108 & 21 & 0.6 \\
\hline
\end{tabular}

Figure 3 shows retention cascade of Delhi for period from March 2013 through April 2014. The retention of patients at 4 steps of the cascade after excluding deaths, transfer outs, treatment stopped due to medical reasons was $84 \%, 88 \%, 83 \%$ and $89.5 \%$, respectively. Compared to overall cascade of the last decade (April 2004 to March 2014), there is improvement at all steps of cascade, except step 2 showing decline in CD4-testing by 4.4\%. "On ART” and "Pre-ART” LTFU rates declined to 9.4\% and 7.4\%, respectively.

\section{Discussion}

NACO established 8 ART centres in various medical colleges/government hospitals of country including 2 in Delhi in 2004. The ART programme was scaled up during NACP-III by setting up 425 ART centres in country. About 1,758,748 patients were enrolled in HIV-care in last decade. However, only about 768,840 (62.5\%), out of 1,230,144 patients ever initiated ART, are currently alive [2], while status of many patients in pre-ART is unknown. 
Retention Cascade (2013-2014)

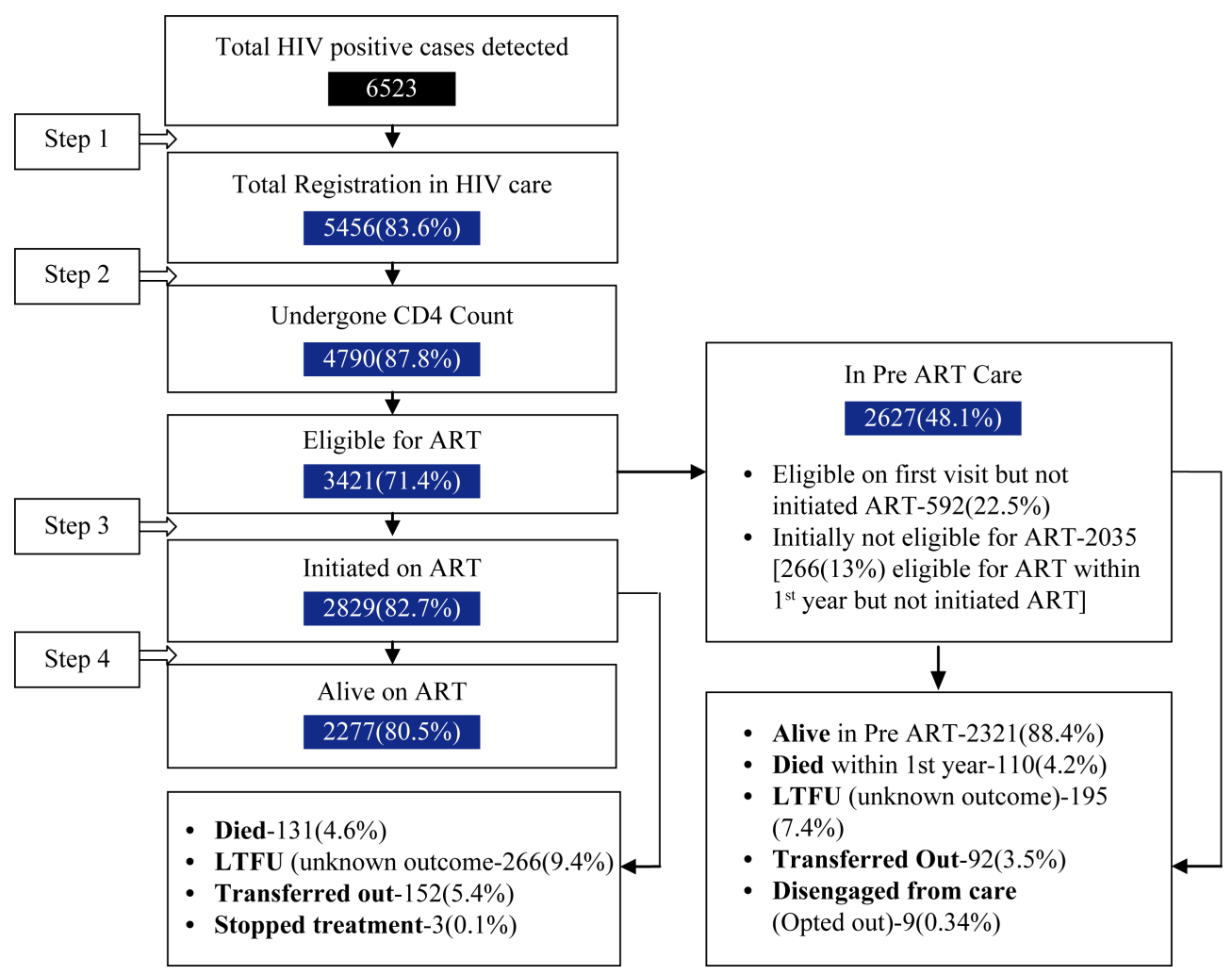

Figure 3. Retention cascade of Delhi in 5th year of NACP-III (April 2013-March 2014).

The UNAIDS has set new target named "90-90-90" to increase to $90 \%$ first, the proportion of people living with HIV who know their diagnosis, second, the proportion of people living with HIV receiving antiretroviral treatment, and third, the proportion of people on HIV treatment who have an undetectable viral load [7]. There is no published report on decadal retention cascade of country. However, analysis of retention cascade of Delhi for last decade showed attrition of patients at all steps of cascade with high attrition at step 1 and step 3 . In a cohort study from Andhra Pradesh, $18.4 \%$ people diagnosed with HIV did not enter into care and only $31.4 \%$ followed all stages of care [8]. The limitation of present study is that cohort data has not been examined to evaluate retention in HIV-care. Also, viral load assessment is not a routine component of treatment cascade being a resource limited country.

The retention of patients in pre-ART care was 3 times lower than those initiated ART. There is no published report on overall outcome of patients in pre-ART care for country because ART programme in India have traditionally focused more on patients remaining in care after initiation of ART than on earlier stages of care. In cohort study from Andhra Pradesh, attrition was higher in pre-ART stages than after ART initiation [8]. In a study in Malawi, attrition rates were 31 times higher among patients not started on ART compared with those started on ART [9]. Other studies on pre-ART period have demonstrated significant patient attrition rates with only $20 \%-33 \%$ of those identified as HIV-positive retained in pre-ART care to initiate ART [10]. ILT drive was not successful in tracking 97\% pre-ART LTFU patients due to incomplete addresses/or migration because before 2009 address proof of patients on enrolment into HIV care was not mandatory. With retrieval of 45 patients in HIV-care, ILT has helped in determining baseline information about patients currently in active pre-ART care and patients alive on ART to be followed up during NACP-IV instead of earlier huge numbers in pre-ART care. This may facilitate better follow-up of patients currently on ART and in active pre-ART care in NACP-IV. It will also allow attention on recent LTFU cases which are more likely to be tracked. A pre-ART tracking study in Uganda revealed that $31 \%$ had died, $37 \%$ had disengaged from care, with $63 \%$ being retained in care elsewhere [11].

Several factors may be responsible for the attrition of patients at each level of retention cascade [12]. One 
factor for attrition of patients from HIV-care in Delhi is migration. ART services in northern India were first rolled out in Delhi. Eventually PLHIV from neighboring states enrolled for HIV-care in Delhi. After start of services in their states, many PLHIV may have silently transferred to respective states. About a quarter of patients diagnosed with HIV did not enroll in HIV-care. This may be due to unavailability of HIV test results on same day, denial of positive-status, inadequate post-test counseling and lack of adequate coordination between ICTCs \& ART centres. About 8\% patients did not undergo CD4-test probably due to non-availability of CD4 machines in 5/9 ART centres which were linked to other ART centers and CD4-test was done twice a week. Hence patients had to wait for test \& result. About a quarter of patients eligible for ART could not be initiated treatment. This may be due to non acceptability of lifelong treatment, non disclosure, fear of side effects of ARV medicines, and belief in alternative system of medicine. About a quarter of patients initiated ART did not return for second visit after 15 days-1 month of initiating treatment. This probably reflects poor treatment education and lack of initial monitoring of adverse effects of ARV medicines. About 20\% patients were LTFU after 2 years of initiation of ART. Some of them may have stopped treatment on symptomatic improvement, or shifted to alternate system of medicine or migrated to native state or died. Tracking patients through home visits by ORWs is challenging in view of potential risk of disclosure, denial by some patients, and few ORWs deployed to track many cases.

During NACP-III [13] [14], various strategies such as co-location of ICTC and ART centers, improving quality of post-test counseling, treatment education, free laboratory tests, monitoring adverse effects of drugs, reducing frequency of visits, SMS/telephonic call to patient missing visit, optimizing treatment regimens, electronic transfer of patient to other ART centre, reducing stigma \& discrimination in hospitals, roll out of postexposure prophylaxis helpline for health providers, community engagement, and outreach to track LTFU cases through DAPCUs resulted in improvement at all stages of retention cascade in 2013-14 except at step 2. This was due to interruption in supply of CD4 kits in February, 2014. However, despite various measures undertaken [13] [14], desired 90\% continuum of care at 4 steps of the cascade could not be achieved in 2013-14. This suggests need to identify measures to improve continuum of care. Several studies suggest that various good practices can improve linkage to care [3].

An important strategy to track patients in HIV-care is PLHIV Smart Card/unique identifier [12] [15]. NACO planned to roll out Smart Card project in Delhi and 6 high prevalence states in 2009 to ensure uninterrupted access to treatment for migrants \& people travelling for short durations, avoid duplicate registrations across the country, and facilitate overall monitoring of ART programme. However, the project is waiting for roll out of Strategic Information Management System (SIMS) at ART centers.

One reason for attrition of patients from HIV-care after initiation of treatment is transport cost and loss of income [1]. In this respect DSACS rolled out a financial assistance scheme for PLHIV on ART in April, 2012 [16]. Among 1530 beneficiaries of the scheme, adherence to treatment has been $100 \%$ except 16 who died. However, major constraint of the scheme is requirement of proof of residence for preceding 3 years and annual family income $(<$ INR 1 lakh) certificate.

Reducing frequency of visits in stable patients may be another strategy to reduce burden of travel cost \& income loss on patients and decongest HIV-care facilities. An innovative patient led strategy was piloted in Mozambique. Amongst group of 6 stable PLHIV only one individual was required to visit ART clinic every month to get medicines for self \& others to reduce workload at health facility [17].

Point of Care (POC) CD4-testing may also help to reduce frequency of visits of the patients and enhance retention in HIV-care [3] [18]. India is piloting POC CD4-testing at 16 ART centers this year.

Devoting adequate time during pre-ART and follow up drug adherence counseling has a potential to improve retention of patients in HIV-care [19]. However, despite deputing extra manpower at the ART centers on basis of patient load during NACP-III, quality of counseling continues to be an issue because of attrition of qualified and trained manpower due to meager remuneration structure of NACO, resulting in break in counselor-patient rapport and continuum of care.

As per national guidelines ART can be initiated only at standalone ART centers which has following advantages: first; delivering services through skilled manpower, second; easy to monitor few centres, and third; adherence to treatment is monitored closely. However, infrastructure and manpower of ART centers, which was sufficient initially, is not currently so due to enrolment of new patients year by year. Further, limited numbers of service delivery points have potential for break in continuum of care due to travel distance, loss of income, and inadequate counseling due to overcrowding. Hence WHO strategy of decentralization and task shifting by inte- 
gration of standalone ART services with primary level services, district level hospitals, antenatal care, MNCH, TB and harm reduction programmes need to be adapted to improve access to care locally by increasing entry points to HIV-care [1] [3]. In Lesotho [20] and Cameroon [21], ART delivery at decentralized level had a positive impact on PLHIV's quality of life and well being.

Considering huge losses in pre-ART care, WHO recommendations of initiating ART at CD4 threshold of 500 cells/cu.mm or less may facilitate retaining patients in HIV-care to save lives, improve clinical outcomes and reduce HIV incidence [3].

\section{Conclusion}

To conclude despite several measures taken in last decade of ART programme, retention of patients in HIV-care is still a challenge and suggests need to adopt strategies such as POC CD4-testing, PLHIV tracking through Smart Card/Unique ID, decreasing frequency of visits of stable patients, adopting new WHO strategy of initiating ART at CD4-count cut-off level of 500 cells/cum.mm to reduce number of patients in Pre-ART care, decentralizing standalone ART services by integrating HIV-care in primary care facilities, district level hospitals, antenatal clinics, and TB programme.

\section{References}

[1] Ulett, K.B., Willig, J.H., Lin, H.-Y., et al. (2009) The Therapeutic Implications of Timely Linkage and Early Retention in HIV Care. AIDS Patient Care STDS, 23, 41-49. http://dx.doi.org/10.1089/apc.2008.0132

[2] National AIDS Control Organization (2014) Journey of ART Programme in India—Story of a Decade. http://naco.gov.in/upload/Final\%20Publications/2014/Journey\%20of\%20ART\%20Programme\%20in\%20India\%20-\%2 0Final.pdf

[3] WHO (2013) Consolidated Guidelines on the Use of Antiretroviral Drugs for Treating and Preventing HIV Infection. http://www.who.int/hiv/pub/guidelines/arv2013/download/en/

[4] National AIDS Control Organisation (2010) HIV Sentinel Surveillance 2010-11, a Technical Brief. http://www.naco.gov.in/upload/Publication/M\&E\%20Surveillance,\%20Research-Technical\%20Report\%20on\%20HIV \%20Estimates\%202010.PDF

[5] National AIDS Control Organisation (2013) Antiretroviral Therapy Guidelines for HIV Infected Adults and Adolescents.

http://www.naco.gov.in/upload/Policies\%20\&\%20Guidelines/Antiretroviral\%20Therapy\%20Guidelines\%20for\%20HI V-Infected\%20Adults\%20and\%20Adolescents.pdf

[6] Department of AIDS Control, Ministry of Health \& Family Welfare, Government of India (2013) Updated Guidelines for Prevention of Parent-to-Child Transmission (PPTCT) of HIV Using Multi Drug Anti-Retroviral Regimen in India. New Delhi.

[7] UNAIDS (2014) New Targets Set for 2020 for Reducing the HIV Epidemic in Latin America and the Caribbean. http://www.unaids.org/en/resources/presscentre/featurestories/2014/june/20140606lact argets/

[8] Alvarez-Uria, G., Pakam, R., Midde, M. and Naik, P.K. (2013) Entry, Retention, and Virological Suppression in an HIV Cohort Study in India: Description of the Cascade of Care and Implications for Reducing HIV-Related Mortality in Low- and Middle-Income Countries. Interdisciplinary Perspectives on Infectious Diseases, 2013, Article ID: 384805. http://dx.doi.org/10.1155/2013/384805

[9] Taylor-Smith, K., Zachariah, R. and Massaquoi, M. (2010) Unacceptable Attrition among WHO Stages 1 and 2 Patients in a Hospital-Based Setting in Rural Malawi: Can We Retain Such Patients within General Health System? Transactions of the Royal Society of Tropical Medicine and Hygiene, 104, 313-319. http://dx.doi:10.1016/j.trstmh.2010.01.007

[10] Rosen, S., Fox, M.P. and Gill, C.J. (2007) Patient Retention in Antiretroviral Therapy Programs in Sub-Saharan Africa: A Systematic Review. PLoS Medicine, 4, e298. http://dx.doi:10.1371/journal.pmed.0040298

[11] Geng, E.H., Bangsberg, D.R., Musinguzi, N., et al. (2010) Understanding Reasons for and Outcomes of Patients Lost to Follow-Up in Antiretroviral Therapy Programs in Africa through a Sampling-Based Approach. Journal of Acquired Immune Deficiency Syndromes, 53, 405-411. http://dx.doi:10.1097/QAI.0b013e3181b843f0

[12] WHO (2011) Retention in HIV Programmes_-Defining the Challenges and Identifying Solutions. Meeting Report, Geneva. http://whqlibdoc.who.int/publications/2012/9789241503686 eng.pdf

[13] National AIDS Control Organisation (2007) Antiretroviral Therapy Guidelines for HIV Infected Adults and Adolescents including Post-Exposure. 
http://nacoonline.org/upload/Policies\%20\&\%20Guidelines/1.\%20Antiretroviral\%20Therapy\%20Guidelines\%20for\%2 0HIV-Infected\%20Adults\%20an\%20Adolescents\%20Including\%20Post-exposure.pdf

[14] National AIDS Control Organization (2011) Care, Support \& Treatment. Phase III, India. http://www.naco.gov.in/upload/IEC\%20Division/Parliamentarian\%20Forum\%204-5\%20july\%202011/ART\%20Mono graph.pdf

[15] UNAIDS (2009) Developing and Using Individual Identifiers for the Provision of Health Services including HIVProceedings from a Workshop Montreux, Switzerland.

http://www.unaids.org/en/media/unaids/contentassets/documents/dataanalysis/20110520 Unique Identifiers Meeting Report_Montreux.pdf

[16] Gupta, A.K., Rawat, N., Rai, K., Rana, S. and Chakraborty, S. (2013) Orphan and Vulnerable Children Infected or Affected by HIV/AIDS in Delhi-Situational Analysis and State Government's Initiative of Household Economic Strengthening. Vulnerable Children and Youth Studies, 8, 161-170. http://dx.doi.org/10.1080/17450128.2012.738949

[17] Decroo, T., Telfer, B., Biot, M., et al. (2011) Distribution of Antiretroviral Treatment through Self-Forming Groups of Patients in Tete Province, Mozambique. Journal of Acquired Immune Deficiency Syndromes, 56, 39-44. http://dx.doi:10.1097/QAI.0b013e3182055138.

[18] Larson, B., Schnippel, K., Ndibongo, B., et al. (2012) How to Estimate the Cost of Point-of-Care CD4-Testing in Program Settings: An Example Using the Alere Pima ${ }^{\mathrm{TM}}$ Analyzer in South Africa. PLoS ONE, 7, e35444. http://dx.doi:10.1371/journal.pone.0035444

[19] Gardner, L.I., Giordano, T.P., Marks, G., et al. (2014) Enhanced Personal Contact with HIV Patients Improves Retention in Primary Care: A Randomized Trial in 6 US HIV Clinics. Clinical Infectious Diseases, 59, 725-734. http://dx.doi:10.1093/cid/ciu357

[20] Cohen, R., Lynch, S., Bygrave, H., et al. (2009) Antiretroviral Treatment Outcomes from a Nurse-Driven, Community-Supported HIV/AIDS Treatment Programme in Rural Lesotho: Observational Cohort Assessment at Two Years. Journal of the International AIDS Society, 12, 23. http://dx.doi:10.1186/1758-2652-12-23

[21] Boyer, S., Protopopescu, C., Marcellin, F., et al. (2012) Performance of HIV-Care Decentralization from the Patient's Perspective: Health-Related Quality of Life and Perceived Quality of Services in Cameroon. Health Policy Plan, 27, 301-315. http://dx.doi:10.1093/heapol/czr039 
Scientific Research Publishing (SCIRP) is one of the largest Open Access journal publishers. It is currently publishing more than 200 open access, online, peer-reviewed journals covering a wide range of academic disciplines. SCIRP serves the worldwide academic communities and contributes to the progress and application of science with its publication.

Other selected journals from SCIRP are listed as below. Submit your manuscript to us via either submit@scirp.org or Online Submission Portal.
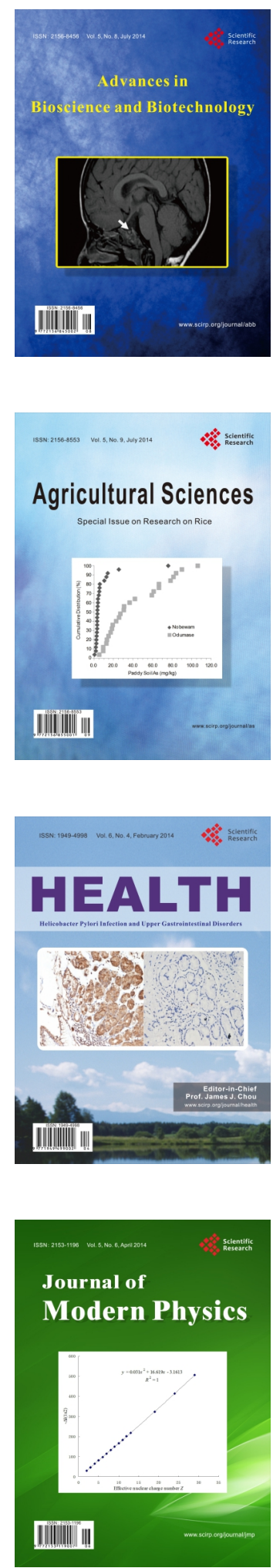
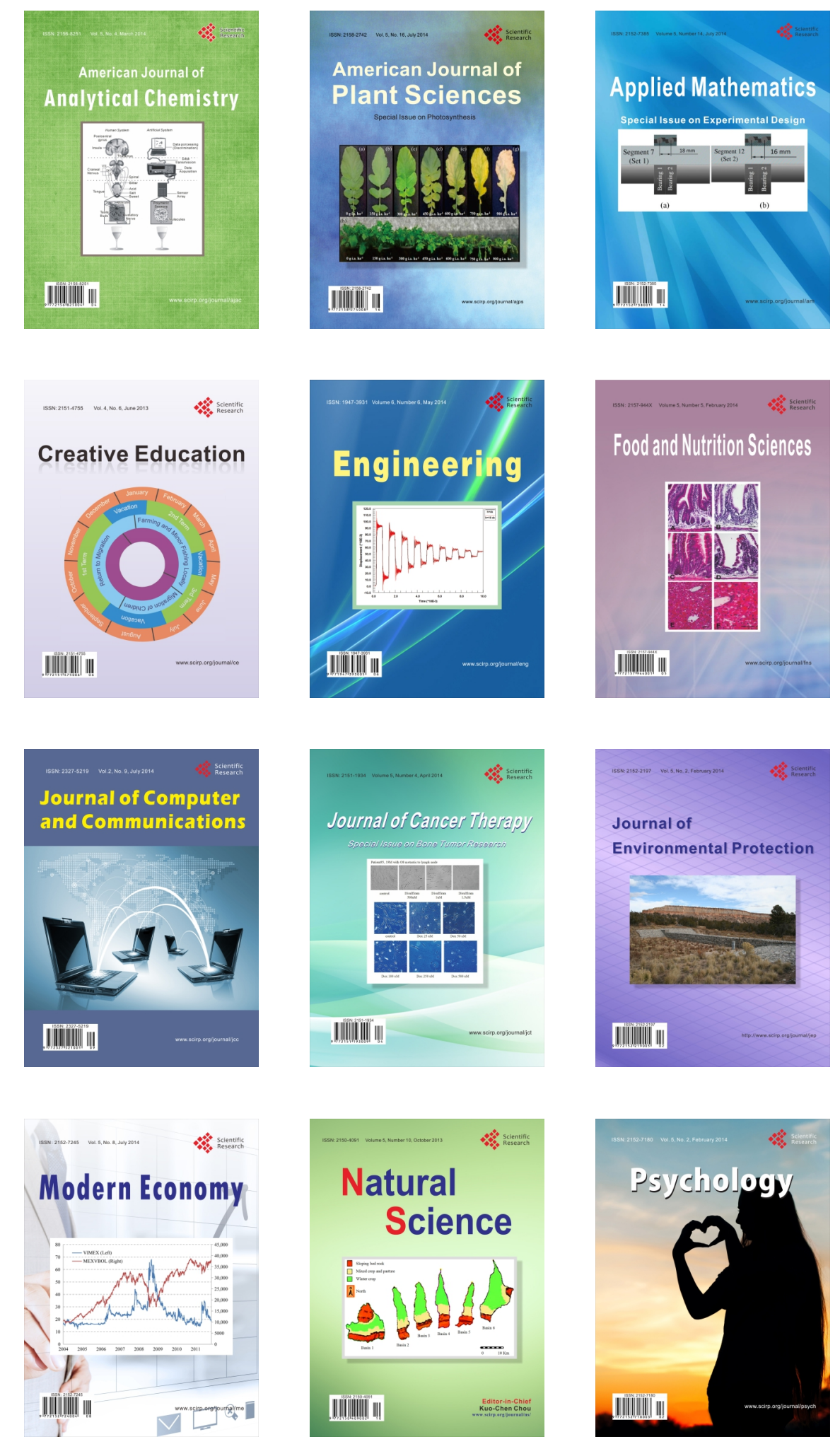